Annals of Pure and Applied Mathematics

Vol. 15, No. 1, 2017, 97-104

ISSN: 2279-087X (P), 2279-0888(online)

Published on 11 December 2017

www.researchmathsci.org

DOI: http://dx.doi.org/10.22457/apam.v15n1a9

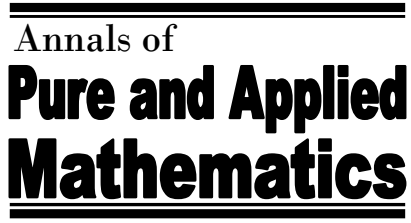

\title{
On Regular Complex Neutrosophic Graphs
}

\author{
P. Thirunavukarasu ${ }^{1}$ and $R$. Suresh ${ }^{2}$ \\ ${ }^{1}$ P.G \& Research Department of Mathematics \\ Periyar E.V.R College (Autonomous), Tiruchirappalli - 620023 \\ Tamilnadu, India. Email: ptavinash1967@gmail.com \\ ${ }^{2}$ Department of Mathematics \\ Kings College of Engineering, Pudukkottai (Dt) - 613303 \\ Tamilnadu, India. Email: sureshnational@gmail.com
}

Received 5 November 2017; accepted 4 December 2017

\begin{abstract}
In this paper, we introduced a new concept of complex neutrosophic graphs called regular complex neutrosophic graph with degree for complex neutrosophic graphs and studied some properties of this new concept. The concept of regular complex neutrosophic graph is an extension of complex neutrosophic fuzzy graphs of type.
\end{abstract}

Keywords: Complex fuzzy set, Fuzzy graph, Complex fuzzy graph, Neutrosophic fuzzy graph, Regular complex neutrosophic graph.

\section{AMS Mathematics Subject Classification (2010): $05 \mathrm{C} 72$}

\section{Introduction}

Fuzzy set was introduced by Zadeh [10] whose basic component is only a membership function. In Zadeh's fuzzy set, the sum of membership degree and a non-membership degree is equal to one. Complex fuzzy set (CFS) [6]-[7] is a new development in the theory of fuzzy systems in [10]. The concept of CFS is an extension of fuzzy set, by which the membership for each element of a complex fuzzy set is extended to complexvalued state. The first definition of fuzzy graphs was proposed by Kauffmann [2] in 1973, from the Zadeh's fuzzy relations [10,11,12]. The first definition and applications of a complex fuzzy graph defined in [1,9].In this paper, we planned to extend our theoretical concepts of complex fuzzy graph into regular complex neutrosophic fuzzy graph.

In this paper, Section 1 describes about introduction and informative collection of existing concepts about complex fuzzy set and fuzzy graph. Definition of complex fuzzy set, complex fuzzy graph discussed in section 2 . In section 3, new and innovative concept of complex neutrosophic fuzzy set, complex neutrosophic graph, and introduced degree of complex neutrosophic graph and regular complex neutrosophic graph with example and its applications are discussed. Concluded of this paper in section 4.

\section{Complex fuzzy sets}

In a complex fuzzy set, membership values are complex numbers in the unit disc of the complex plane [6]-[7]. Although the introductory theory of the CFS has been presented [6], the research on complex fuzzy system designs and applications using the concept of 


\section{P. Thirunavukarasu and R. Suresh}

CFS is found rarely. Since the seminal paper in 1965 by Zadeh proposed Fuzzy Sets [10], a huge amount of literature has appeared on different aspects of fuzzy sets and their applications [10-12].

Definition 2.1. Ramot et al. [6] proposed an important extension of these ideas, the Complex Fuzzy Sets (CFS), where the membership function of a CFS is complex-valued, different from fuzzy complex numbers developed in Buckley et.al. The membership function to characterize a CFS consists of an amplitude function and a phase function. In other words, the membership of a CFS is in the two-dimensional complex-valued unit disc space, instead of in the one-dimensional real-valued unit interval space. Thus, CFS can be much richer in membership description than traditional fuzzy set.

Assume there is a complex fuzzy set $S$ whose membership function $\mu_{S}(h)$ is given as follows,

$$
\begin{aligned}
\mu_{S}(h)=r_{S}(h) e^{j \omega_{S}(h)} & =\operatorname{Re}\left(\mu_{S}(h)\right)+j \operatorname{Im}\left(\mu_{S}(h)\right) \\
& =r_{S}(h) \cos \left(\omega_{S}(h)\right)+j r_{S}(h) \sin \left(\omega_{S}(h)\right)
\end{aligned}
$$

where $j=\sqrt{-1}, h$ is the base variable for the complex fuzzy set, $r_{s}(h)$ is the amplitude function of the complex membership, $\omega_{\mathrm{s}}(h)$ is the phase function of the complex membership function.

The property of sinusoidal waves appears obviously in the definition of complex fuzzy set. In the case that $\omega_{s}(h)$ equals to 0 , a traditional fuzzy set is regarded as a special case of a complex fuzzy set.

Definition 2.2. Let V be a nonempty set. A fuzzy graph is a pair of functions $\mathrm{G}:(\sigma, \mu)$ where $\sigma$ is a fuzzy subset of $\mathrm{V}$ and $\mu$ is a symmetric fuzzy relation on $\sigma$. i.e. $\sigma: \mathrm{V} \rightarrow[0,1]$ and $\mu: \mathrm{VxV} \rightarrow[0,1]$ such that $\mu(\mathrm{u}, \mathrm{v}) \leq \sigma(\mathrm{u}) \wedge \sigma(\mathrm{v})$ for all $\mathrm{u}, \mathrm{v}$ in $\mathrm{V}$.

Definition 2.3. [1] A complex fuzzy directed graph $G$ is a quadruple of the form $\mathrm{G}=(\mathrm{V}, \sigma, \mathrm{E}, \phi)$, where $\mathrm{V}$ is a complex fuzzy set referred to as the set of vertices and $\mathrm{E}$ $\subseteq V X V$ is a complex fuzzy set of edges. $\sigma: V \rightarrow[0,1] X[0,1]$ is a mapping from $\mathrm{V}$ to $[0,1] X[0,1]$. i.e, $\sigma$ is the assignment of the complex degrees of membership to members of $\mathrm{V}, \varphi: E \rightarrow[0,1] X[0,1]$ is a function that maps elements of the form e $\in E:(u, v)$ to $[0,1] X[0,1]$, where $\mathrm{u} \in V, v \in V$, we assume that $\mathrm{V} \cap \mathrm{E}=\phi$. In general, we use the form $\mathrm{e}=(\mathrm{a}, \mathrm{b})$ to denote a specific edge that is said to connect the vertices a and $\mathrm{b}$. For an undirected graph both $\mathrm{e}_{1}=(\mathrm{u}, \mathrm{v})$ and $\mathrm{e}_{2}=(\mathrm{v}, \mathrm{u})$ are in the domain of $\varphi$. 
On Regular Complex Neutrosophic Graphs

Example 2.4.

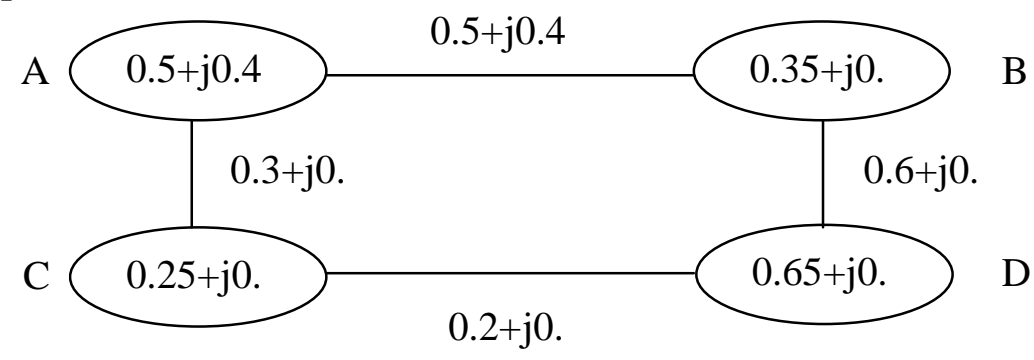

Figure 2.1. Complex fuzzy graph

Definition 2.5. Alternative definition of complex fuzzy graph given as follows. An complex fuzzy graph is defined as $\mathrm{CG}=(\mathrm{V}, \mathrm{E}, r, \omega)$ where $\mathrm{V}$ is the set of vertices and $\mathrm{E}$ is the set of edges. $r$ represents a amplitude membership function defined on $\mathrm{VxV}$ and $\omega$ represents a phase membership function defined on $\mathrm{VxV}$. We denote $r\left(v_{i}, v_{j}\right)$ by $r_{i j}$ and $\omega\left(v_{i}, v_{j}\right)$ by $\omega_{i j}$ such that (i) $0 \leq r_{i j}, \omega_{i j} \leq 1$. Hence $(\mathrm{VxV}, r, \omega)$ is an complex fuzzy set

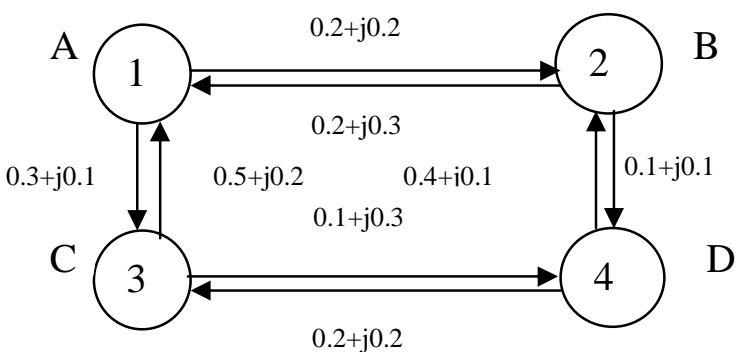

Figure 2.2. Directed complex fuzzy graph

\section{Regular complex neutrosophic graph}

\section{Definition 3.1. Complex neutrosophic set}

A complex neutrosophic set as defined in [3], i.e. A complex neutrosophic set defined on a universe of discourse $\mathrm{X}$, which is charaterized by a truth membership function $T_{s}(x)$ ,an indeterminacy membership function $I_{s}(x)$, and a falsity membership function $F_{s}(x)$ that assigns a complex-valued grade of $T_{s}(x), I_{s}(x)$, and $F_{s}(x)$ in $\mathrm{S}$ for any $x \in X$.

The values $T_{s}(x), I_{s}(x), F_{s}(x)$ and their sum may all within the unit circle in the complex plane and so is of the following form, $T_{s}(x)=p_{S}(x) e^{j \mu_{S}(x)}$, $I_{s}(x)=q_{S}(x) e^{j \vartheta_{S}(x)} \quad$ and $\quad F_{s}(x)=r_{S}(x) e^{j \omega_{S}(x)}$ where $\quad p_{S}(x), q_{S}(x), r_{S}(x)$ and $\mu_{S}(x), v_{S}(x), \omega_{S}(x)$ are, amplitude term and phase term of $T_{s}(x), I_{s}(x), F_{s}(x)$ respectively, real valued and $p_{S}(x), q_{S}(x), r_{S}(x) \in[0,1]$ such that 
P. Thirunavukarasu and R. Suresh

${ }^{-} 0 \leq p_{S}(x)+q_{S}(x)+r_{S}(x) \leq 3^{+}$. The complex neutrosophic set $\mathrm{S}$ can be represented in set form as $S=\left\{\left(x, T_{S}(x)=a_{r}, I_{S}(x)=a_{t}, F_{S}(x)=a_{F}\right): x \in X\right\}$,

Where, $T_{S}: X \rightarrow\left\{a_{T}: a_{T} \in C,\left|a_{T}\right| \leq 1\right\}, I_{S}: X \rightarrow\left\{a_{I}: a_{I} \in C,\left|a_{I}\right| \leq 1\right\}$,

$F_{S}: X \rightarrow\left\{a_{F}: a_{F} \in C,\left|a_{F}\right| \leq 1\right\}$, and $\left|T_{S}+I_{S}+F_{S}\right| \leq 3$.

\section{Definition 3.2. [8] Complex neutrosophic graph}

Let $\mathrm{V}$ be a non-void set. Two functions are considered as follows: $\rho=\left(\rho_{T}, \rho_{I}, \rho_{F}\right): V \rightarrow[0,1]^{3}$ and $\omega=\left(\omega_{T}, \omega_{I}, \omega_{F}\right): V \times V \rightarrow[0,1]^{3}$. We suppose $A=\left\{\left(\rho_{T}(x), \rho_{T}(y)\right) \mid \omega_{T}(x, y) \geq 0\right\}$,

$B=\left\{\left(\rho_{I}(x), \rho_{I}(y)\right) \mid \omega_{I}(x, y) \geq 0\right\}, C=\left\{\left(\rho_{F}(x), \rho_{F}(y)\right) \mid \omega_{F}(x, y) \geq 0\right\}$,

we have considered $\omega_{T}, \omega_{I}$ and $\omega_{F} \geq 0$ for all set A,B,C, since it is possible to have edge degree $=0$ (for $\mathrm{T}$, or $\mathrm{I}$, or $\mathrm{F})$. The triad $(V, \rho, \omega)$ is defined to be complex neutrosophic graph of type 1 (CNG1) if there are functions $\alpha: A \rightarrow[0,1], \beta: B \rightarrow[0,1]$ and $\delta: C \rightarrow[0,1]$

such that $\omega_{T}(x, y)=\alpha\left(\rho_{T}(x), \rho_{T}(y)\right), \omega_{I}(x, y)=\beta\left(\rho_{I}(x), \rho_{I}(y)\right)$ and $\omega_{F}(x, y)=\delta\left(\rho_{F}(x), \rho_{F}(y)\right), x, y \in V$, here $\rho(x)=\left(\rho_{T}(x), \rho_{I}(x), \rho_{F}(x)\right), x \in V$ are the complex truth membership, complex indeterminate-membership and complex false-membership of the vertex $\mathrm{x}$ and $\omega(x, y)=\left(\omega_{T}(x, y), \omega_{I}(x, y), \omega_{F}(x, y)\right), x, y \in V$ are the complex truth membership, complex indeterminate-membership and complex false-membership values of the edge $(\mathrm{x}, \mathrm{y})$.

\section{Example 3.3.}

\begin{tabular}{|c|c|c|c|c|}
\hline $\begin{array}{c}\text { Membership } \\
\text { function of } \\
\text { Vertex set }\end{array}$ & $\mathrm{A}$ & $\mathrm{b}$ & $\mathrm{c}$ & $\mathrm{d}$ \\
\hline$\rho_{T}$ & $0.3 e^{j \frac{2 \pi}{3}}$ & $0.4 e^{j \frac{\pi}{2}}$ & $0.2 e^{j \frac{\pi}{3}}$ & $0.6 e^{\frac{j \pi}{3}}$ \\
\hline$\rho_{I}$ & $0.2 e^{j \frac{4 \pi}{3}}$ & $0.8 e^{j \frac{\pi}{3}}$ & $0.7 e^{j \frac{\pi}{4}}$ & $0.5 e^{j \frac{\pi}{3}}$ \\
\hline$\rho_{F}$ & $0.5 e^{j \frac{\pi}{3}}$ & $0.3 e^{j \frac{2 \pi}{3}}$ & $0.6 e^{j \frac{3 \pi}{4}}$ & $0.8 e^{j \frac{\pi}{4}}$ \\
\hline
\end{tabular}

Table 3.1: Complex truth-membership, complex indeterminate-membership and complex false-membership of the vertex set.

Here,

$$
A=\left\{\left(0.3 e^{j \frac{2 \pi}{3}}, 0.4 e^{j \frac{\pi}{2}}\right),\left(0.3 e^{j \frac{2 \pi}{3}}, 0.2 e^{j \frac{\pi}{3}}\right),\left(0.4 e^{j \frac{\pi}{2}}, 0.6 e^{j \frac{\pi}{3}}\right),\left(0.4 e^{j \frac{\pi}{2}}, 0.6 e^{j \frac{\pi}{3}}\right)\right\}
$$


On Regular Complex Neutrosophic Graphs

$$
\begin{gathered}
B=\left\{\left(0.2 e^{j \frac{4 \pi}{3}}, 0.8 e^{j \frac{\pi}{3}}\right),\left(0.2 e^{j \frac{4 \pi}{3}}, 0.7 e^{j \frac{\pi}{4}}\right),\left(0.8 e^{j \frac{\pi}{3}}, 0.5 e^{j \frac{\pi}{3}}\right),\left(0.8 e^{j \frac{\pi}{3}}, 0.7 e^{j \frac{\pi}{4}}\right)\right\}, \\
C=\left\{\left(0.5 e^{j \frac{\pi}{3}}, 0.3 e^{j \frac{2 \pi}{3}}\right),\left(0.5 e^{j \frac{\pi}{3}}, 0.6 e^{j \frac{3 \pi}{4}}\right),\left(0.3 e^{j \frac{2 \pi}{3}}, 0.8 e^{j \frac{\pi}{4}}\right),\left(0.3 e^{j \frac{2 \pi}{3}}, 0.6 e^{j \frac{3 \pi}{4}}\right)\right\}
\end{gathered}
$$

Then,

\begin{tabular}{|c|c|c|c|c|}
\hline $\begin{array}{c}\text { Membership function } \\
\text { of edge set }\end{array}$ & $(\mathrm{a}, \mathrm{b})$ & $(\mathrm{a}, \mathrm{c})$ & $(\mathrm{b}, \mathrm{d})$ & $(\mathrm{b}, \mathrm{c})$ \\
\hline$\omega_{T}$ & $0.4 e^{j \frac{2 \pi}{3}}$ & $0.4 e^{j \frac{\pi}{3}}$ & $0.6 e^{j \frac{\pi}{2}}$ & $0.4 e^{j^{\frac{\pi}{2}}}$ \\
\hline$\omega_{I}$ & $0.8 e^{j \frac{4 \pi}{3}}$ & $0.7 e^{j \frac{4 \pi}{3}}$ & $0.8 e^{j \frac{\pi}{3}}$ & $0.8 e^{j \frac{\pi}{3}}$ \\
\hline$\omega_{F}$ & $0.5 e^{j \frac{2 \pi}{3}}$ & $0.3 e^{j \frac{2 \pi}{3}}$ & $0.6 e^{j \frac{3 \pi}{4}}$ & $0.6 e^{j \frac{3 \pi}{4}}$ \\
\hline
\end{tabular}

Table 3.2: Complex truth-membership, complex indeterminate-membership and complex false-membership of the edge set.

Then, the corresponding complex neutrosophic graph is shown in the following figure.

a

b

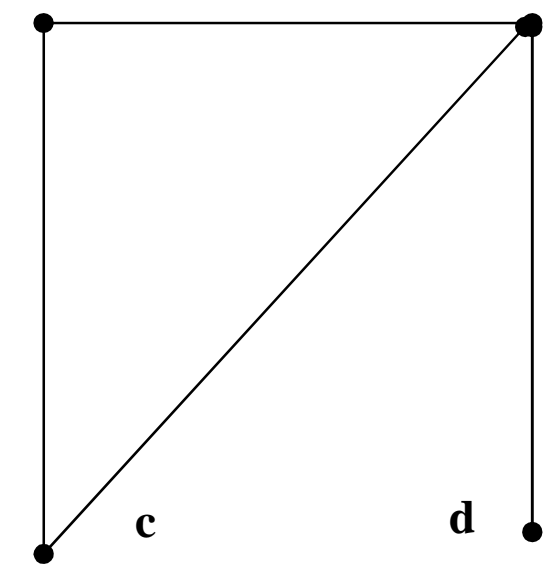

Figure 3.1: Complex neutrosophic graph

$\left(\rho_{T}(a), \rho_{I}(a), \rho_{F}(a)\right)=\left(\omega_{T}(a, b), \omega_{I}(a, b), \omega_{F}(a, b)\right)=\left(\rho_{T}(b), \rho_{I}(b), \rho_{F}(b)\right)=$ $\left(0.3 e^{j \frac{2 \pi}{3}}, 0.2 e^{j \frac{4 \pi}{3}}, 0.5 e^{j \frac{\pi}{3}}\right) \quad\left(0.4 e^{j \frac{2 \pi}{3}} 0.8 e^{j \frac{4 \pi}{3}} 0.5 e^{j \frac{2 \pi}{3}}\right) \quad\left(0.4 e^{j \frac{\pi}{2}}, 0.8 e^{j \frac{\pi}{3}}, 0.3 e^{j \frac{2 \pi}{3}}\right)$

$\left(\omega_{T}(a, c), \omega_{I}(a, c), \omega_{F}(a, c)\right)=\left(\omega_{T}(b, c), \omega_{1}(b, c), \omega_{F}(b, c)\right)=\left(\omega_{T}(b, d), \omega_{1}(b, d), \omega_{F}(b, d)\right)=$ $\left(0.4 e^{j \frac{\pi}{3}}, 0.7 e^{j \frac{4 \pi}{3}}, 0.3 e^{j \frac{2 \pi}{3}}\right) \quad\left(0.4 e^{j \frac{\pi}{2}}, 0.8 e^{j \frac{\pi}{3}}, 0.6 e^{j \frac{3 \pi}{4}}\right) \quad\left(0.6 e^{j \frac{\pi}{2}}, 0.8 e^{j \frac{\pi}{3}}, 0.6 e^{j \frac{3 \pi}{4}}\right)$ 
P. Thirunavukarasu and R. Suresh

$$
\begin{aligned}
& \left(\rho_{T}(c), \rho_{I}(c), \rho_{F}(c)\right)=\left(\rho_{T}(d), \rho_{I}(d), \rho_{F}(d)\right)=\left(0.2 e^{j \frac{\pi}{3}}, 0.7 e^{j \frac{\pi}{4}}, 0.6 e^{j \frac{3 \pi}{4}}\right) \\
& \left(0.6 e^{\frac{j \pi}{3}}, 0.5 e^{j \frac{\pi}{3}}, 0.8 e^{j \frac{\pi}{4}}\right)
\end{aligned}
$$

Definition 3.4. The degree of a vertex in complex neutrosophic graph defined as in [4]. Let $G=(\rho, \omega)$ be complex neutrosophic graph. The degree of a vertex x is denoted by

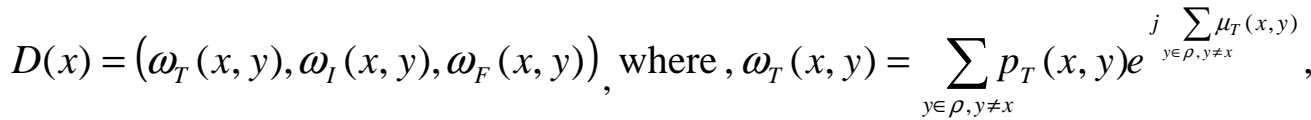

$$
\begin{aligned}
& \omega_{I}(x, y)=\sum_{y \in \rho, y \neq x} q_{I}(x, y) e^{j \sum_{y \in \rho, y \neq x} v_{I}(x, y)}, \text { And } \omega_{F}(x, y)=\sum_{y \in \rho, y \neq x} r_{F}(x, y) e^{j \sum_{y \in \rho, y \neq x} \omega_{F}(x, y)} .
\end{aligned}
$$

Definition 3.5. A complex neutrosophic graph $G=(\rho, \omega)$ is called a regular complex neutrosophic graph of degree $\left(m_{1}, m_{2}, m_{3}\right)$ if $D_{G}(S)=\left(m_{1}, m_{2}, m_{3}\right), \forall S \in V$, where V is Vertex set. i.e., the regularity of a complex neutrosophic graph means that the degrees of each vertex is same. But, for large networks, it is difficult to confirm that all vertices have same degree. If major number of vertices have the same degree or almost same degree, then the graph is defined to be regular.

Example 3.6. Consider the graph $G=(\rho, \omega)$ such that $\rho=\{a, b, c, d\}$ and $\omega=\{(a, b),(a, c),(c, d),(b, d)\}$. The corresponding regular complex neutrosophic graph $G=(\rho, \omega)$ is given below.

a

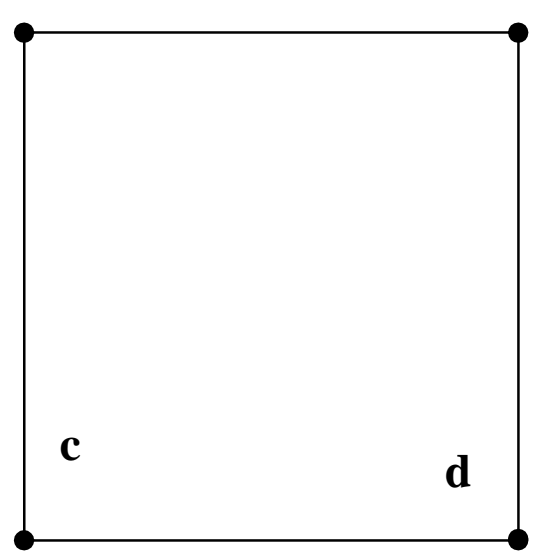

b

Figure 3.2: Regular complex neutrosophic graph g

$$
\begin{aligned}
& \left(\rho_{T}(a), \rho_{I}(a), \rho_{F}(a)\right)=\left(\omega_{T}(a, b), \omega_{I}(a, b), \omega_{F}(a, b)\right)=\left(\rho_{T}(b), \rho_{I}(b), \rho_{F}(b)\right)= \\
& \left(0.3 e^{j \frac{2 \pi}{3}}, 0.2 e^{j \frac{4 \pi}{3}}, 0.5 e^{j \frac{\pi}{3}}\right) \quad\left(0.2 e^{j \frac{2 \pi}{3}} 0.8 e^{j \frac{4 \pi}{3}} 0.5 e^{j \frac{2 \pi}{3}}\right) \quad\left(0.4 e^{j \frac{\pi}{2}}, 0.8 e^{j \frac{\pi}{3}}, 0.3 e^{j \frac{2 \pi}{3}}\right)
\end{aligned}
$$


On Regular Complex Neutrosophic Graphs

$$
\begin{aligned}
& \left(\omega_{T}(a, c), \omega_{I}(a, c), \omega_{F}(a, c)\right)=\left(\omega_{F}(b, d), \omega_{1}(b, d), \omega_{F}(b, d)\right)=\left(0.6 e^{j \frac{\pi}{3}}, 0.7 e^{j \frac{4 \pi}{3}}, 0.3 e^{j \frac{2 \pi}{3}}\right) \\
& \left(0.6 e^{j \frac{\pi}{3}}, 0.7 e^{j \frac{4 \pi}{3}}, 0.3 e^{j \frac{2 \pi}{3}}\right) \\
& \left(\rho_{T}(c), \rho_{I}(c), \rho_{F}(c)\right)=\left(\omega_{F}(b, c), \omega_{1}(b, c), \omega_{F}(b, c)\right)=\left(\rho_{T}(d), \rho_{I}(d), \rho_{F}(d)\right)= \\
& \left(0.2 e^{j \frac{\pi}{3}}, 0.7 e^{j \frac{\pi}{4}}, 0.6 e^{j \frac{3 \pi}{4}}\right)\left(0.2 e^{j \frac{2 \pi}{3}} 0.8 e^{j \frac{4 \pi}{3}} 0.5 e^{j \frac{2 \pi}{3}}\right)\left(0.6 e^{\frac{j \pi}{3}}, 0.5 e^{j \frac{\pi}{3}}, 0.8 e^{j \frac{\pi}{4}}\right)
\end{aligned}
$$

\section{Conclusion}

An extended form of neutrosophic graph is presented in this paper, so-called regular complex neutrosophic graph. Complex neutrosophic set can handle the redundant nature of uncertainty, incompleteness, indeterminacy, inconsistency, etc. A complex neutrosophic set is defined by a complex-valued truth membership function, complexvalued indeterminate membership function, and a complex-valued falsehood membership function. Therefore, a complex-valued truth membership function is a combination of traditional truth membership function with the additional term is called phase term. Thus, in this way, the truth amplitude term represents uncertainty and the phase term represents periodicity in the uncertainty. Thus, a complex-valued truth membership function represents uncertainty with periodicity as a whole. This paper is an introductory paper of regular complex neutrosophic graph, and indeed, much research is still needed for the full comprehension of order, degree, size and matrix representation of regular complex neutrosophic graph. The regular complex neutrosophic graph presented in this paper is an entire general concept which is not limited to a specific application.

\section{REFERENCES}

1. Dan E. Tamir, Naphtali D. Rishe, Mark Last and Abraham Kandel, Soft Computing Based Epidemical Crisis Prediction, Springer International, Publishing Switzerland 2015, R.R. Yager et al. (eds.), Intelligent Methods for Cyber Warfare, Studies in Computational Intelligence 563, 2015.

2. A.Kauffman, Introduction a la theorie des sous ensembles Flous, Masson, vol. 1. (1973).

3. M.Ali and F.Smarandache, Complex neutrosophic set, Neural Computing \& Applications, DOI 10.1007/s00521-015-2154-y.

4. A.Nagoor Gani and S.Shajitha Begum, Degree, order and size in intuitionistic fuzzy graphs, International Journal of Algorithms, Computing and Mathematics, 3(3) (2010) 11-16.

5. B.V.Praba, V.M.Chandrasekaran and G.Deepa, Energy of an intuitionistic fuzzy graph, Italian Journal of Pure and Applied Mathematics, 32 (2014) 431-444.

6. D.Ramot, R.Milo, M.Friedman and A.Kandel, Complex fuzzy sets, IEEETrans. Fuzzy Syst., 10(2) (2002) 171-186.

7. D.Ramot, M.Friedman, G.Langholz and A.Kandel, Complex fuzzy logic, IEEE Trans Fuzzy Syst., 11(4) (2003) 450- 461.

8. S.Broumi, M.Talea, A.Bakali and F.Smarandache, Complex neutrosophic graphs of type 1, IEEE Publication, 978-1-5090-5795-5/17 @2017 IEEE. 


\section{P. Thirunavukarasu and R. Suresh}

9. P.Thirunavukarasu, R.Suresh and K.K.Viswanathan, Energy of a complex fuzzy graph, International J.of Math. Sci. \&Engg. Appls., 10(I) (2016).

10. L.A.Zadeh, Fuzzy sets, Information and Control, 8 (1965) 338-353.

11. L.A.Zadeh, Similarity relations and fuzzy ordering, Information Aciences, 3 (1971) 177-200.

12. L.A.Zadeh, Is there a need for fuzzy logic? Information Sciences, 178 (2008) 2751- 2779. 